\title{
Analysis of selected anthropometric parameters in infants in relation to the risk of developing hip dysplasia
}

\author{
Aleksander Szwed, Dawid Ciechanowicz, Piotr Modliborski, Sławomir Zacha, Daniel Kotrych, \\ Bartosz Kruk, Adam Kamiński \\ Department of Paediatric Orthopaedics and Oncology, Pomeranian Medical University, Szczecin, Poland
}

\section{ABSTRACT}

\begin{abstract}
Aim: Despite the known risk factors for the occurrence of $\mathrm{DDH}$, such as the breech presentation, we still do not know all the factors that determine the more frequent incidence of dysplasia in female children. The aim of the study was compare anthropometric parameters of boys and girls in the first months of life, and to relate these measurements to cases of children diagnosed with developmental hip dysplasia (DDH).

Material and methods: 502 children were enrolled in the study - 273 (54.4\%) girls and 229 (45.6\%) boys. The mean age at the time of the examination was: $1.8 \pm 0.9$ months, boys; $1.98 \pm 1.4$ months girls. The statistical analysis covered the birth weight, body weight at the time of the examination, length of pregnancy, type of delivery and pelvic measurements.

Results: In the study, we observed a higher frequency of DDH in the group of girls: 15 cases in 13 (4.76\%) girls. We observed relationship ( $p=0.023322$ ) between the week of pregnancy at time of delivery and the incidence of DDH. Pelvic dimensions did not show such a relationship. The group of girls with dysplasia $(n=13)$ differed from the group of healthy girls in terms of the week of pregnancy $(p=0.040014)$ and in terms of birth weight $(p=0.048079)$.

Conclusions: Boys and girls do not differed significantly in terms of pelvic width and hip spacing. The hypothesis that it is the differences in the structure of the pelvis that may contribute to the more frequent incidence of DDH in girls is not confirmed.
\end{abstract}

\section{KEY WORDS:}

anthropometry, measures, developmental hip dysplasia, pelvic dimensions, children's orthopedics.

\section{INTRODUCTION}

Developmental dysplasia of the hip (DDH) is the most frequently diagnosed disease of the musculoskeletal system in newborns and infants. It applies to $1-3 \%$ of newborn and is responsible for $30 \%$ of primary arthroplasty procedures in patients below 60 years of age $[1,2]$. Undiagnosed and untreated DDH may, in extreme cases, lead to complete dislocation of the hip joint. Therefore, early diagnosis and treatment is so important in the prevention of complications of DDH.
The etiology of the disease is not entirely clear. Over the last decades, a number of risk factors for DDH have been identified. The well known risk factors include: female sex, high fetal weight, breech presentation, positive family history of DDH or oligohydramnios [3-6]. DDH is also more common in children with other 'tightness' syndromes, such as congenital foot defects or congenital torticollis $[1,7]$. However, one of the main risk factors for $\mathrm{DDH}$ is the female gender of the fetus (ratio girls : boys $9: 1$ ) [1]. The reason why DDH is more common in female children is not fully understood. Until recently, this

\section{ADDRESS FOR CORRESPONDENCE:}

Dawid Ciechanowicz, Department of Paediatric Orthopaedics and Oncology, Pomeranian Medical University,

Unii Lubelskiej 1, 71-252 Szczecin, Poland, e-mail: dawid.ciechanowicz@gmail.com 
predilection was considered to be related to the hormonal differences between male and female [1]. It was widely believed that higher levels of estrogen in female fetuses were a factor that increased the risk of DDH [8]. Recent reports, however, have shown that there is no specific difference in the level of hormones in fetuses diagnosed with $\mathrm{DDH}$ and those with properly developed joints [9-13]. In connection with the results of the above-mentioned studies on hormonal differences in newborns and the predisposition of female fetuses to develop $\mathrm{DDH}$, aspects that take into account mechanical factors, predisposing to the development of dysplasia, may become increasingly important. Therefore, the assumption that the differences in the pelvic structure of female fetuses are a factor increasing the risk of developing hip dysplasia may be justified.

Theory of 'packing disorder' suggests that tight constrictive conditions in the uterus can lead to malposition of the hips, and subsequent DDH, especially in fetuses in the breech position $[14,15]$. The mechanical nature of the defect is in this case undeniable, resulting from incorrect pressure on the hip joint at the time of its maturation and differentiation, leading to the development of hip dysplasia. The pelvis, which is the widest element of the bone and joint system of a newborn, is subjected to compressive forces, which in effect lead to distortion of the development of the hip joint.

It can therefore be presumed that it is the anatomical differences in the pelvic structure that predispose some female fetuses to the development of DDH.

The aim of the study is to compare anthropometric parameters of boys and girls in the first months of life, and to relate these measurements to cases of children diagnosed with developmental hip dysplasia.

\section{MATERIAL AND METHODS}

To the retrospective study 502 patients were included, 229 of whom were boys (45.6\%) and 273 (54.4\%) were girls. All children were screened for developmental dysplasia of the hip (DDH).

The data from the gynecological interview were analyzed (i.e. the week of pregnancy at the time of delivery (Hbd), the way of delivery, the child's birth weight) and the family history of developmental hip dysplasia.

During the visit, each child underwent a full clinical examination for Developmental Dysplasia of the Hip Joint, as well as ultrasound examination of the hip joints according to the Graf's methodology. The examination was performed using an Ultrasound Scanner - ESAOTE AAU5 (802-260-017) [16].

Developmental Dysplasia of the Hip Joint was found in the case of abnormalities in the clinical examination AND ultrasound examination. In the ultrasound examination, the norm was found when the angle $\alpha>60^{\circ}$ and the angle $\beta>55^{\circ}$ (according to the Graf method) $[17,18]$.
Additionally, pelvic dimensions were examined in all children. The distantia trochanterica was measured between the greater trochanter of the femurs in the child's supine position. The distantia spinarum was measured as the width contained between the anterior superior iliac spines in the child's supine position. During the same visit, the child's weight was measured and recorded.

All data were statistically processed using the STATISTICA 13. After checking the data distribution of the obtained results, the $t$-test was performed for independent samples in order to compare the differences in the pelvic dimensions, birth weight and body weight at the time of the clinical examination (current body weight), in the group of boys and the group of girls. Logistic regression was performed to investigate the relationship between week of pregnancy at time of delivery, pelvic dimensions, body weight at the time of examination, and birth weight, and the occurrence of hip dysplasia. $P<0.05$ was considered to be statistically significant.

\section{RESULTS}

The mean age at the time of the examination was 1.98 \pm 1.35 months in the group of girls, while in the group of boys $1.78 \pm 0.89$ months.

In both groups, we observed a similar mean gestation week at the time of delivery (Hbd) - boys 38,63 \pm 1.8 ; girls $38,85 \pm 1.7$. The vast majority of pregnancies were ended between 38 and 40 weeks - 442 children (88.05\%). Preterm deliveries were recorded in 60 cases $(11.95 \%)-$ boys 32 (13.97\%); girls 28 (10.26\%). The mean birth weight in the group of boys was 3366,820 \pm 548.9 grams, while in the group of girls it was $3248,033 \pm 547.3$ grams. The girls birth weight was statistically significantly lower compared to the boys group by $3.53 \%(p=0.019368)$ (Table 1). Children in both groups were most often born by vaginal birth: boys 121 (52.84\%); girls 273 (61.54\%).

In the group of boys, the mean body weight at the time of the examination (current body weight) was $5027,346 \pm 1011,76$ grams, while in the group of girls it was $4750.963 \pm 974.1$ grams. Thus, the weight at the time of the examination in the group of girls was statistically significantly lower by $5.5 \%$ compared to boys $(p=0.002795)$ (Table 1).

The mean distantia spinarum was: boys $-11.49 \pm 8.49$; girls: $10.94 \pm 1.24$. The mean distantia trochanterica was: boys: $8.84 \pm 0.76$; girls: $8.75 \pm 0.70$. The boys showed larger pelvic dimensions by $4.77 \%(p=0.292970)$ and $1.02 \%$ $(p=0.222461)$ (Table 1$)$.

In our study, we observed a higher incidence of hip dysplasia in the group of girls: 15 cases in 13 (4.76\%) girls (two cases were diagnosed with dysplasia in both hips), compared to boys: $1(0.4 \%)$ case.

The performed logistic regression showed a statistically significant relationship ( $p=0.023322)$ between the week of pregnancy at time of delivery and the incidence 
TABLE 1. Comparison of data from the gynecological interview and data from the examination between a group of boys and a group of girls. Data marked in red indicate statistically significant changes $(p<0.05)$

\begin{tabular}{|l|c|c|c|c|}
\hline & $\begin{array}{c}\text { Group of boys }(n=229) \\
\text { Mean } \pm \text { SD }\end{array}$ & $\begin{array}{c}\text { Group of girls }(n=273) \\
\text { Mean } \pm \text { SD }\end{array}$ & Difference [\%] & P-value \\
\hline Age (months) & $1.780349 \pm 0.89$ & $1.978755 \pm 1.35$ & $10.03 \%$ & 0.058366 \\
\hline Hbd (week) & $38.63277 \pm 1.8$ & $38.84536 \pm 1.7$ & $0.55 \%$ & 0.307560 \\
\hline Birth weight (grams) & $3366.820 \pm 548.9$ & $3248.033 \pm 547.3$ & $3.53 \%$ & 0.019368 \\
\hline Current body weight (grams) & $5027.346 \pm 1011.76$ & $4750.963 \pm 974.1$ & $5.5 \%$ & 0.002795 \\
\hline Distantia spinarum (cm) & $11.48725 \pm 8.49$ & $10.93985 \pm 1.24$ & $4.77 \%$ & 0.292970 \\
\hline Distantia trochanterica $(\mathrm{cm})$ & $8.836000 \pm 0.76$ & $8.746114 \pm 0.70$ & $1.02 \%$ & 0.222461 \\
\hline
\end{tabular}

TABLE 2. Comparison of pelvic dimensions and birth weight; current weight and week of delivery $(\mathrm{Hbd})$ in the group of girls diagnosed with $\mathrm{DDH}$ and in the group of healthy girls. Data marked in red indicate statistically significant changes $(p<0.05)$

\begin{tabular}{|l|c|c|c|c|}
\hline & $\begin{array}{c}\text { Group of girls with DDH } \\
(n=13) \\
\text { Mean } \pm \text { SD }\end{array}$ & $\begin{array}{c}\text { Group of healthy girls } \\
(n=260) \\
\text { Mean } \pm \text { SD }\end{array}$ & Difference [\%] & P-value \\
\hline Hbd (week) & $40.00000 \pm 1.09$ & $38.78689 \pm 2.09$ & $3.03 \% *$ & 0.040014 \\
\hline Birth weight (grams) & $3532.000 \pm 477.09$ & $3233.559 \pm 572.55$ & $8.45 \%$ & 0.048079 \\
\hline Current body weight (grams) & $4852.000 \pm 770.03$ & $4745.992 \pm 1045.58$ & $2.18 \%$ & 0.698755 \\
\hline Distantia spinarum (cm) & $11.32714 \pm 1.73$ & $10.92166 \pm 1.22$ & $3.58 \%$ & 0.237334 \\
\hline Distantia trochanterica (cm) & $8.841667 \pm 0.62$ & $8.741343 \pm 0.71$ & $1.13 \%$ & 0.629718 \\
\hline
\end{tabular}

* Girls with diagnosed dysplasia were born statistically 1.2 weeks later than healthy girls

of hip dysplasia. Pelvic dimensions did not show such a relationship in the studied groups - the distantia spinarum $(p=0.697567)$, the distantia trochanterica $(p=0.913690)$. Also, the birth weight $(p=0.585653)$ and the current body weight at the time of the examination $(p=0.492021)$ did not show such a relationship.

The group of girls with dysplasia $(n=13)$ differed statistically significantly from the group of healthy girls in terms of the week of pregnancy at time of delivery $(p=0.040014)$ and in terms of birth weight $(p=0.048079)$. Namely, girls diagnosed with hip dysplasia had a slightly higher birth weight on average by $8.45 \%$ and were born later ( 40 week vs. 38,79 week) compared to girls in the healthy group. Additionally, the group of girls with dysplasia had a larger distantia spinarum on average by $3.58 \%(p=0.237334)$ and a larger distantia trochanterica on average by $1.13 \%(p=0.629718)$ (Table 2$)$.

\section{DISCUSION}

Recent reports clearly indicate that there is a difference in the structure of the pelvis in primates $[19,20]$. Sexual dimorphism is confirmed for adults and is believed to be related to the role of females in giving birth to offspring [21]. This difference in the structure of adult pelvis has not been confirmed so far in the study of immature animals. There is a high probability that the differentiation of the structure and dimorphism in the pelvis takes place during the puberty period under the influence of sex hormones. There are single reports of differences in the structure of the skeleton of immature people in the area of the femur, humerus and mandible [22]. However, so far, no gender difference in pelvic structure has been proven in children.

Our research raises several important issues. To the best of our knowledge, this is the first study to compare the pelvic dimensions of male and female babies. It is also the first report comparing the pelvic dimensions of children diagnosed with DDH with those born with normal hip joints. However, the study has a number of limitations. The dimensions of the pelvis were assessed only in a clinical study, assessing only two dimensions: the distantia spinarum and the distantia trochanterica. Additionally, the study included a group of 502 infants in which 16 cases of DDH were found in 14 children (13 girls, 1 boy). The number of examined children may be insufficient to show differences in the structure of the pelvis in different sex groups, especially in the group of children diagnosed with dysplasia.

In our study, the group of boys had slightly larger pelvic dimensions, however, we found no statistically significant differences in the width of the pelvis itself and the width of the hip joints spacing. Despite the fact that girls had DDH much more often, it was not confirmed that the pelvis of sick girls differed statistically in terms of the width and spacing of the hip joints in relation to the dimensions of the pelvis in children with normal hip joints. This contradicts the hypothesis of the study in which we assumed that the pelvic dimensions of girls should be larger than that of boys at birth, which would 
explain the higher incidence of dysplasia in female fetuses. The test results, however, turned out to be opposite to the expectations, and it was boys in the first weeks of life that showed wider pelvis dimensions than girls. This difference was not statistically significant and may be the result of higher birth weight for boys than for girls. Nevertheless, the hypothesis of anatomical differences in pelvic structure depending on sex in newborn children was not confirmed in the study.

Fetal weight over $4000 \mathrm{~g}$ has been recognized as risk factors for DDH, and its influence on the development of dysplasia has been proven in numerous studies [23-25]. However, our study did not fully confirm this relationship. The boys in the study were born with a higher birth weight than the girls, but their weight did not contribute to the higher incidence of hip dysplasia. Only the coincidence of two risk factors, i.e. the female sex and higher birth weight, had an impact on the risk of DDH. It was confirmed in our study that in girls with a higher birth weight, hip dysplasia was more common. The authors of the studies who assessed the ultrasound parameters of the hip joints in relation to commonly recognized DDH risk factors have reached similar conclusions. The studies have shown that only the coexistence of two risk factors, i.e. greater body weight and female sex, increases the risk of abnormalities in the ultrasound examination of the hip joints in newborns [26-28].

However, the above relationship was not confirmed for another parameter, namely the dimensions of the pelvis. The pelvis of children diagnosed with hip dysplasia did not differ significantly in relation to the pelvic dimensions of healthy children. There was also no evidence of a positive correlation between female gender and pelvic width and the risk of DDH. In this case, the assumption that larger pelvic dimensions in female fetuses have an influence on the more frequent incidence of hip dysplasia has not been confirmed.

Undoubtedly, the effect of "packaging disorders" is of great importance in initiating changes leading to the development of DDH $[15,29]$. Incorrect positioning of the pelvis, limited space for the development of hip joints are undeniable factors contributing to the development of dysplasia [28]. The possibility of sexual dimorphism as an aspect responsible for the more frequent incidence of dysplasia in girls should be taken into account. The research on these differences should be extended to include radiological examinations of the dimensions of the pelvis and hip joints in newborns and infants. Only a standardized, broad and in-depth analysis of pelvic dimensions in a group of children in similar age can give an answer about possible differences in its structure.

\section{CONCLUSIONS}

Our study does not indicate that boys and girls differed statistically significantly in terms of pelvic width and hip spacing. Thus, the hypothesis that it is the differences in the structure of the pelvis that may contribute to the more frequent incidence of dysplasia in girls is not confirmed here.

The pathogenesis of DDH is multifactorial, therefore further research looking for a relationship in the group of girls diagnosed with dysplasia is recommended.

\section{DISCLOSURE}

The authors declare no conflict of interest.

\section{REFERENCES}

1. Agarwal A, Gupta N. Risk factors and diagnosis of developmental dysplasia of hip in children. J Clin Orthop Trauma 2012; 3: 10-14.

2. Shaw BA, Segal LS; Section on Orthopaedics. Evaluation and Referral for Developmental Dysplasia of the Hip in Infants. Pediatrics 2016; 138: e20163107.

3. de Hundt M, Vlemmix F, Bais JM, et al. Risk factors for developmental dysplasia of the hip: a meta-analysis. Eur J Obstet Gynecol Reprod Biol 2012; 165: 8-17.

4. Woodacre T, Ball T, Cox P. Epidemiology of developmental dysplasia of the hip within the UK: refining the risk factors. J Child Orthop 2016; 10: 633-642.

5. Manoukian D, Rehm A. Oligohydramnios: should it be considered a risk factor for developmental dysplasia of the hip?. J Pediatr Orthop B 2019; 28: 442-445.

6. Vafaee AR, Baghdadi T, Baghdadi A, Jamnani RK. DDH Epidemiology Revisited: Do We Need New Strategies? Arch Bone Jt Surg 2017; 5: 440-442.

7. Kural B, Devecioğlu Karapınar E, Yılmazbaş P, et al. Risk Factor Assessment and a Ten-Year Experience of DDH Screening in a WellChild Population. Biomed Res Int 2019; 2019: 7213681.

8. Alsaleem M, Set KK, Saadeh L. Developmental Dysplasia of Hip: A Review. Clin Pediatr (Phila) 2015; 54: 921-928.

9. Vogel I, Andersson JE, Uldbjerg N. Serum relaxin in the newborn is not a marker of neonatal hip instability. J Pediatr Orthop 1998; 18: 535-537.

10. Thieme WT, Wynne-Davies R. Clinical examination and urinary oestrogen assays in newborn children with congenital dislocation of the hip. J Bone Joint Surg Br 1968; 50: 546-550.

11. Rhodes AM, Clarke NM. A review of environmental factors implicated in human developmental dysplasia of the hip. J Child Orthop 2014; 8: 375-379.

12. Andersson JE, Vogel I, Uldbjerg N. Serum 17 beta-estradiol in newborn and neonatal hip instability. J Pediatr Orthop 2002; 22: 88-91.

13. Forst J, Forst C, Forst R, Heller KD. Pathogenetic relevance of the pregnancy hormone relaxin to inborn hip instability. Arch Orthop Trauma Surg 1997; 116: 209-212.

14. Ortiz-Neira CL, Paolucci EO, Donnon T. A meta-analysis of common risk factors associated with the diagnosis of developmental dysplasia of the hip in newborns. Eur J Radiol 2012; 81: e344-e351.

15. Shefelbine SJ, Carter DR. Mechanobiological predictions of growth front morphology in developmental hip dysplasia. J Orthop Res 2004; 22: 346-352.

16. Joiner ER, Andras LM, Skaggs DL. Screening for hip dysplasia in congenital muscular torticollis: is physical exam enough? J Child Orthop 2014; 8: 115-119.

17. Graf $\mathrm{R}$. The diagnosis of congenital hip-joint dislocation by the ultrasonic Combound treatment. Arch Orthop Trauma Surg 1980; 97: 117-133. 
18. Schams M, Labruyère R, Zuse A, Walensi M. Diagnosing developmental dysplasia of the hip using the Graf ultrasound method: risk and protective factor analysis in 11,820 universally screened newborns. Eur J Pediatr 2017; 176: 1193-1200.

19. Tague RG. Pelvic sexual dimorphism among species monomorphic in body size: relationship to relative newborn body mass. J Mammal 2016; 97: 503-517.

20. Zollikofer CP, Scherrer M, Ponce de León MS. Development of Pelvic Sexual Dimorphism in Hylobatids: Testing the Obstetric Constraints Hypothesis. Anat Rec (Hoboken) 2017; 300: 859-869.

21. Leong A. Sexual dimorphism of the pelvic architecture: a struggling response to destructive and parsimonious forces by natural \& mate selection. Mcgill J Med 2006; 9: 61-66.

22. Carlson KJ, Grine FE, Pearson OM. Robusticity and sexual dimorphism in the postcranium of modern hunter-gatherers from Australia. Am J Phys Anthropol 2007; 134: 9-23.

23. von Deimling U, Brähler JM, Niesen M, et al. Effect of birth weight on hip maturation in the newborn infant. Klin Padiatr 1998; 210: 115-119.

24. Bower C, Stanley FJ, Kricker A. Congenital dislocation of the hip in Western Australia. A comparison of neonatally and postneonatally diagnosed cases. Clin Orthop Relat Res 1987; 224: 37-44.

25. Roposch A, Protopapa E, Malaga-Shaw O, et al. Predicting developmental dysplasia of the hip in at-risk newborns. BMC Musculoskelet Disord 2020; 21: 442.

26. Chan A, McCaul KA, Cundy PJ, et al. Perinatal risk factors for developmental dysplasia of the hip. Arch Dis Child Fetal Neonatal Ed 1997; 76: F94-F100.

27. Orak MM, Karaman O, Gursoy T, et al. Effect of increase in birth weight in a newborn on hip ultrasonography. J Pediatr Orthop B 2015; 24: 507-510.

28. Ömeroğlu H, Akceylan A, Köse N. Associations between risk factors and developmental dysplasia of the hip and ultrasonographic hip type: a retrospective case control study. J Child Orthop 2019; 13: 161-166.

29. Verbruggen SW, Kainz B, Shelmerdine SC, et al. Altered biomechanical stimulation of the developing hip joint in presence of hip dysplasia risk factors. J Biomech 2018; 78: 1-9. 